\title{
Maximizing Throughput When Achieving Time Fairness in Multi-Rate Wireless LANs
}

\author{
Yuan Le*, Liran $\mathrm{Ma}^{\dagger}$, Wei Cheng*, Xiuzhen Cheng*, Biao Chen ${ }^{\ddagger}$ \\ *Department of Computer Science, The George Washington University, Washington DC, USA \\ ${ }^{\dagger}$ Department of Computer Science, Texas Christian University, Fort Worth, TX, USA \\ $\ddagger$ Department of Computer \& Information Science, University of Macau, Macau, China \\ Email: ppzt@gwmail.gwu.edu, 1.ma@tcu.edu, wcheng@gwu.edu, cheng@gwu.edu, bchen@umac.mo
}

\begin{abstract}
This paper focuses on designing a distributed medium access control algorithm that aims at achieving time fairness among contending stations and throughput maximization in an 802.11 wireless LAN. The core idea of our proposed algorithm lies in that each station needs to select an appropriate contention window size so as to fairly share the channel occupancy time and maximize the throughput under the time fairness constraint. The derivation of the proper contention window size is presented rigorously. We evaluate the performance of our proposed algorithm through an extensive simulation study, and the evaluation results demonstrate that our proposed algorithm leads to nearly perfect time fairness, high throughput, and low collision overhead.
\end{abstract}

\section{INTRODUCTION}

A fundamental problem in 802.11 wireless LANs is to design Medium Access Control (MAC) algorithms for sharing network resources among contending stations. The primary objective is to fully utilize all available resources (such as channel access opportunity or channel occupancy time) while maintaining a certain "fairness" in the allocations concerning different stations. There exist four types of popular fairness criteria: throughput fairness, time fairness, max-min fairness, and proportional fairness. Throughput fairness and time fairness simply try to distribute the resources - throughput or channel occupation time - to stations equally [1]. Max-min fairness and proportional fairness, on the other hand, are defined as optimization problems.

There are many proposed MAC algorithms in the literature that either explicitly or implicitly satisfy one or more types of the four fairness criteria. For example, it is well known that the 802.11 standard [2] employs the Distributed Coordination Function (DCF) as its default medium access control method. It has been shown that DCF provides an equal long-term transmission opportunity to each station in the network [3]. If each station adopts the same frame size, throughput fairness can be achieved.

However, there are multiple bitrates defined in the 802.11 standard so as to adapt to channel condition dynamics. Some studies [4], [5] reveal that in a multi-rate environment, throughput fairness (i.e., the equal transmission opportunity) can severely degrade the overall network performance. The main reason for the performance degradation is due to the fact that the channel is excessively occupied by slow bitrate stations because it takes longer time for them to transmit the same size frame. To remedy this problem, many algorithms that target different types of fairness are proposed. For an instance, Banchs et al. propose a throughput allocation criterion and two allocation schemes that are based on proportional fairness in [6]. Another example is Idle Sense [7] where channel access is regulated by an estimation of the number of idle slots and station bitrates attempting to achieve time fairness and throughput enhancement.

Inspired by these previous work, we propose a novel time fairness based MAC algorithm for a multi-rate wireless LAN. The core idea of our proposed algorithm is that each station needs to select an appropriate contention window size so as to jointly achieve fair sharing of the channel occupancy time and throughput maximization. We rigorously derive the formula to calculate the contention window size. In addition, we evaluate the performance of our algorithm via a comprehensive comparative simulation study. The evaluation results demonstrate that our proposed algorithm possesses the following nice features: i) Channel occupancy time is nearly equally shared among stations; ii) Throughput can be significantly improved under the fairness constraint; iii) Collision overhead is greatly reduced when the network presents a rich bitrate diversity; iv) The optimal content window size can be calculated by each station in a fully distributed manner.

The rest of the paper is organized as follows. Section II discusses the related work. System model is illustrated in Section III. Section IV describes the design of our proposed algorithm. Section V reports our evaluation results and we conclude the paper in Section VI.

\section{RELATED WORK}

There are many studies on the default 802.11 medium access control algorithm (i.e., DCF) [3]-[5], [8]-[10] in the literature. In one of the earlier seminal work [3], Bianchi evaluates the throughput and frame transmission probability of the 802.11 DCF using a Markov chain. In this work, an analytical model with ideal channel conditions is adopted and station bitrates are assumed to be identical. It concludes that 802.11 DCF provides throughput fairness to all stations if they adopt the same frame size. However, because of the rich dynamics of the wireless channel [8], a station needs to transmit at an appropriate bitrate so that the bit error rate can be controlled in an acceptable level. Previous work [4], [5] indicate that in a bitrate diverse environment, algorithms 
that offer equal transmission opportunities can significantly degrade the overall performance.

To address the performance degradation problem, several algorithms are proposed to improve the performance of the default 802.11 DCF by dynamically adjusting the contention window size. A method of estimating the number of active stations using the Kalman filter is proposed in [11]. Based on the estimated number of stations, a suitable contention window size can be calculated. To further improve the performance, Cali et al. derive the average size of the contention window that can maximize the throughput in [12]. With this average contention window size, a distributed algorithm is proposed to enable each station to tune its backoff algorithm at runtime. In [13], stations can exponentially decrease their backoff timer after observing a number of empty slots, and thus the channel utilization is enhanced. Aad et al. propose a simple slow contention window decrease function in [14], in which the contention window size is reduced by half instead of being reset to the initial value after a successful transmission.

Additionally, there exist access control algorithms that are directly based on certain types of fairness such as time fairness and proportional fairness. For example, the long term fairness of DCF is investigated through the conditional probabilities of the number of inter-transmissions in [15]. Another proportional fairness based allocation algorithm is proposed in [6]. In this allocation algorithm, a station sets the initial contention window size inversely proportional to its bitrate. In contrast, a time fairness based resource allocation algorithm is developed in [4]. This algorithm runs on each AP to regulate the frame transmissions. The channel occupation time is equally distributed to each station. However, this algorithm requires a centralized control unit on the AP side, and thus is not adaptive to dynamic environment. Recently, another time fairness based algorithm is devised in [7] where the contention window size is adjusted based on the estimated number of idle slots.

\section{SYSTEM MODEL}

We model a typical 802.11 multi-rate wireless LAN with one AP and $n$ competing stations. Each station is associated to the AP and shares the same channel with other stations. We assume the network is saturated so that each station always has frames to transmit. We assume the following parameters are known constants in our system model: the transmission duration $T_{t}^{i}$ of a station $i$, the average duration of a failure transmission $T_{f}$, and the idle slot duration $T_{s} . T_{t}^{i}$ depends on the bitrate of station $i$ and the average packet size $s_{i}$. For convenience, we further assume that all the stations have the same $s_{i}$. Similarly, $T_{f}$ is represented by the time cost that incurs by a failure transmission. $T_{s}$ is defined by the 802.11 standard.

In our model, we adopt the basic DCF CSMA/CA protocol with no exponential backoff after a failure transmission. We denote the attempt probability $P_{e}^{i}$ of a station $i$ as the probability that station $i$ attempts to transmit a frame. As the stations may have different contention window sizes, the attempt probability $P_{e}^{i}$ can be calculated as in [5]:

$$
P_{e}^{i}=\frac{2}{C W_{i}+1}
$$

where $C W_{i}$ is the contention window size of station $i$.

Consider an event that a station attempts to transmit a frame. The station can successfully transmit the frame if and only if it is the only station that attempts to transmit. Thus, the successful transmission probability $P_{t}^{i}$, the idle probability $P_{i d l e}$ and the transmission failure probability $P_{f}$ can be calculated as:

$$
\begin{aligned}
& P_{t}^{i}=P_{e}^{i} \cdot \prod_{j \neq i}\left(1-P_{e}^{j}\right) \\
& P_{\text {idle }}=\prod_{i=1}^{n}\left(1-P_{e}^{j}\right) \\
& P_{f}=1-\sum_{i=1}^{n} P_{t}^{i}-P_{\text {idle }}
\end{aligned}
$$

In our paper, we adopt a similar throughput definition as that in [3]. Consider a station's transmission in a unit time slot, the expectation of its transmission payload can be expressed as $E(L)=P_{t}^{i} s_{i}$, and the average length of a unit time slot can be calculated by:

$$
E\left(T_{\text {slot }}\right)=\sum_{j=1}^{n}\left(P_{t}^{j} T_{t}^{j}\right)+P_{f} T_{f}+P_{i d l e} T_{s} .
$$

As a result, the throughput of station $i$ can be expressed as the ratio:

$$
W_{i}=\frac{E(L)}{E\left(T_{\text {slot }}\right)}=\frac{P_{t}^{i} s_{i}}{\sum_{j=1}^{n}\left(P_{t}^{j} T_{t}^{j}\right)+P_{f} T_{f}+P_{i d l e} T_{s}} .
$$

And the aggregate throughput is the sum of the per station throughput:

$$
W=\frac{\sum_{i=1}^{n} P_{t}^{i} \cdot s_{i}}{\sum_{i=1}^{n} P_{t}^{i} T_{t}^{i}+P_{f} T_{f}+P_{\text {idle }} T_{s}} .
$$

\section{OUR Algorithm}

The core idea of our proposed algorithm is simple: each station needs to select an appropriate contention window size so as to jointly achieve time fairness and throughput maximization.

\section{A. Analysis of Contention Window Size}

In this section, we illustrate how to compute the appropriate contention window size for each station so as to achieve both time fairness and throughput improvements. Let us start with the time fairness. If any two stations $i$ and $j$ fairly share the channel access time, we have:

$$
T_{t}^{i} P_{t}^{i}=T_{t}^{j} P_{t}^{j}
$$

Combining Eq. (1), Eq. (2) and Eq. (8), we have:

$$
\frac{T_{t}^{i}}{T_{t}^{j}}=\frac{P_{t}^{j}}{P_{t}^{i}}=\frac{P_{e}^{j} \cdot \prod_{k \neq j}\left(1-P_{e}^{k}\right)}{P_{e}^{i} \cdot \prod_{k \neq i}\left(1-P_{e}^{k}\right)}=\frac{C W_{i}-1}{C W_{j}-1} .
$$


Thus, for two arbitrary stations $i$ and $j$ that fairly share the channel access time, their contention window sizes have the following relationship:

$$
C W_{j}=1+\frac{T_{t}^{j}}{T_{t}^{i}}\left(C W_{i}-1\right) .
$$

With this relationship, we now can show how to calculate the appropriate contention window size for each station so as to maximize the aggregate throughput. Recall the aggregate throughput expression in Eq. (7) and notice that $P_{t}^{i} T_{t}^{i}=P_{t}^{j} T_{t}^{j}$ for all $i$ and $j$. Maximizing the aggregate throughput is equivalent to minimize the following cost function:

$$
\begin{aligned}
& \frac{n P_{t}^{i} T_{t}^{i}+P_{f} T_{f}+P_{i d l e} T_{s}}{\sum_{j=1}^{n} P_{t}^{j}} \\
= & \frac{1}{\sum_{j=1}^{n}\left(1 / T_{t}^{j}\right)}-T_{f}+\frac{\left(1-P_{i d l e}\right) T_{f}+P_{i d l e} T_{s}}{P_{t}^{i} T_{t}^{i} \sum_{j=1}^{n}\left(1 / T_{t}^{j}\right)} .
\end{aligned}
$$

In Eq. (11), $T_{t}^{j}, T_{t}^{i}, T_{s}$ and $T_{f}$ are constants for a given network scenario. The variables in Eq. (11) are $P_{t}^{i}, P_{t}^{j}$ and $P_{\text {idle. }}$ Based on Eq. (2) and Eq. (10), $P_{t}^{j}, P_{t}^{i}$ can be expressed as a function of $C W_{i}$. Similarly, $P_{i d l e}$ can also be expressed as a function of $C W_{i}$ according to Eq. (3) and Eq. (10). As a result, the aggregate throughput expression can be expressed as a function of $C W_{i}$. Specifically, minimizing Eq. (11) is equivalent to minimize the following cost function:

$$
\begin{aligned}
& \frac{T_{f}+\left(T_{s}-T_{f}\right) P_{i d l e}}{P_{t}^{i}}=\frac{T_{f}}{P_{t}^{i}}+\left(T_{s}-T_{f}\right) \frac{P_{i d l e}\left(1-P_{e}^{i}\right)}{P_{e}^{i} P_{\text {idle }}} \\
= & \frac{T_{f} \prod_{j=1}^{n}\left[\left(C W_{i}-1\right)+\frac{2 T_{t}^{i}}{T_{t}^{j}}\right]}{2\left(C W_{i}-1\right)^{n-1}}+\left(T_{s}-T_{f}\right) \frac{C W_{i}-1}{2} .
\end{aligned}
$$

Let $\lambda_{j}=\frac{2 T_{t}^{i}}{T_{t}^{j}}$, and $C_{k}$ be:

$C_{k}=\sum_{j_{1}=1}^{n-k+1} \sum_{j_{2}=j_{1}+1}^{n-k+2} \ldots \sum_{j_{k}=j_{k-1}+1}^{n} \prod_{l=1}^{k} \lambda_{j_{l}}, k=1,2,3 \ldots$

After applying $C_{k}$, Eq. (12) can be simplified as follows:

$$
=\frac{T_{s}}{2}\left(C W_{i}-1\right)+\frac{T_{f}}{2} \sum_{k=1}^{n} C_{k}\left(C W_{i}-1\right)^{1-k} .
$$

Next, we show that the minimum value of the cost function (i.e., Eq. (14)) uniquely exists.

Theorem 1: Let $f\left(C W_{i}\right)$ be the function defined by Eq. (14) and Eq. (13), and $T_{t}^{j}, T_{s}$ and $T_{f}$ are parameters defined by the 802.11 standard. The optimal $C W_{i}$ that minimizes the cost function exists uniquely.

Proof: It is clear that $C_{k}>0$ for $k=1,2,3 \ldots$ Consider the first and second derivative of the cost function, we have:

$$
\begin{aligned}
f^{\prime}\left(C W_{i}\right)= & T_{s}-C_{2}\left(C W_{i}-1\right)^{-2}-2 C_{3}\left(C W_{i}-1\right)^{-3} \\
& -\ldots-(n-1)\left(C W_{i}-1\right)^{-n} \\
f^{\prime \prime}\left(C W_{i}\right)= & 2 C_{2}\left(C W_{i}-1\right)^{-3}+6 C_{3}\left(C W_{i}-1\right)^{-4} \\
& +\ldots+n(n-1)\left(C W_{i}-1\right)^{-n-1} .
\end{aligned}
$$

Assume that the maximum bitrate of a station is $54 \mathrm{Mbps}^{1}$ and the minimum bitrate is $1 \mathrm{Mbps}$. We have $T_{t}^{i} / T_{t}^{j} \geq 1 / 54$, for any $i, j=1,2,3 \ldots n$.

Consider $f^{\prime}(2)$ and $\lim f^{\prime}(+\infty)$, we have:

$$
\begin{aligned}
& f^{\prime}(2)=T_{s}-\sum_{k=2}^{n} C_{k} \leq T_{s}-C_{2} \\
& \leq T_{s}-\sum_{j_{1}=1}^{n-1} \sum_{j_{2}=j_{1}+1}^{n} \frac{4\left(T_{t}^{i}\right)^{2}}{T_{t}^{j_{1}} T_{t}^{j_{2}}} \\
& \leq T_{s}-\frac{4\left(T_{t}^{i}\right)^{2}}{T_{t}^{i} T_{t}^{j}} \\
& \leq T_{s}-\frac{2}{27} . \\
& \lim f^{\prime}(+\infty)=T_{s}>0 .
\end{aligned}
$$

Since $T_{s}$ is the duration of a slot time, we have $T_{s} \ll \frac{2}{27}$ and thus $f^{\prime}(2)<0$. Therefore, we can always find a $C W_{o p t} \in$ $[2,+\infty)$ such that $f^{\prime}\left(C W_{o} p t\right)=0$.

In addition, according to Eq. (16), we can see that $f^{\prime \prime}(C W)>0$ for $C W \in[2,+\infty)$. Hence, equation $f^{\prime}(C W)=0$ has a unique solution $C W_{o p t}$ in the range $[2,+\infty)$.

The root of equation $f^{\prime}\left(C W_{i}\right)=0$ uniquely exists. We can apply numerical analysis techniques such as the Newton's method to calculate the numerical value of the optimized contention window size given the values of $T_{t}^{j}, T_{f}$, and $T_{s}$. Via applying the optimized contention window size, each station is bounded to fairly share the channel occupancy time and the aggregate throughput can be significantly improved.

\section{B. Design}

To minimize the implementation overhead in practice, our algorithm design is adopted from the default 802.11 DCF with two major changes. Firstly, each station needs to disable the exponential backoff applied after a failure transmission. Secondly, each station calculates its optimized contention window size based on the cost function listed in Eq. (14).

In order to calculate the optimized contention window size, our algorithm requires each station to know the bitrates of the stations that are within its communication range. Due to the broadcast nature of the wireless medium, a station is able to receive all frames that are within its communication range. As a result, a station can learn the bitrates of its neighboring stations by observing on-going transmissions. The obtained $<$ MAC_address, Bitrate $>$ tuples can be stored and managed in a local table (say Table_t). If a new station arrives or there are changes to the existing tuples, a new contention window size needs to be calculated by calling the contention window calculation function (say $C W_{-} C a l$ ). This $C W_{-}$Cal function can apply the Newton's method to get a numerical solution for minimizing the cost function in Eq. (14).

\footnotetext{
${ }^{1}$ Note that the maximum bitrate depends on the specific 802.11 standard. Here, we assume that the $802.11 \mathrm{~b} / \mathrm{g}$ compatible mode is adopted. A higher maximum bitrate such as $150 \mathrm{Mbps}$ in $802.11 \mathrm{n}$ does not affect the correctness of our proof.
} 


\section{Evaluations}

In this section we evaluate the performance of our proposed algorithm in terms of time fairness, throughput, and collision overhead.

\section{A. Configuration}

We compare the performance of our proposed algorithm with that of the following three widely accepted algorithms: i) The default 802.11 DCF backoff algorithm [2]; ii) The proportional fair throughput allocation algorithm (referred as proportional) proposed in [6]; iii) One of the arguably best time fairness algorithms: Idle Sense [7].

We adopt the Omnet++ and its INET framework as our simulation environment [16]. The INET framework is shipped with the default 802.11 DCF backoff algorithm. We implement the proportional, Idle Sense, and our proposed algorithm. It is worth noting that the implementation of our algorithm is simpler compared to the other two algorithms. In our implementation, the MAC and PHY parameters adopt the values defined in the 802.11 standard. In addition, the packet size is fixed to be 1500 bytes that is the Maximum Transmission Unit (MTU) for Ethernet.

We conduct the performance comparison of the four algorithms in three popular wireless deployment modes: $802.11 \mathrm{~b}$ only, $802.11 \mathrm{~g}$ only, and $802.11 \mathrm{~b} / \mathrm{g}$ compatible. For each type of the deployment modes, there are two types of scenarios adopted. The first type of scenario is introduced in [7] where one slow station competes with $n-1$ fast stations. In contrast, each station in the second type of scenario transmits at a unique bitrate. Hence, the number of stations equals the number of available bitrates of a given mode. The bitrate of each station is set at the initialization phase and remains the same throughout the simulation.

\section{B. Results}

We detail the evaluation results in terms of time fairness, throughput, and collision overhead as follows. All of our results presented are averaged over 10 simulation runs. Each simulation run lasts 20 seconds.

1) Time Fairness: As in Figure 1, we evaluate the shortterm fairness of the channel access time using the Jain's fairness index [1]. We observe that the index of our proposed algorithm consistently approaches 1 in all cases, which closely matches our theoretical analysis in Section IV. We also notice that our proposed algorithm outperforms the other three algorithms in all scenarios. In addition, the fairness improvements are higher when there are more competing stations with different bitrates as shown in Figure 1(d).

2) Throughput: Recall that our objective is to maximize the total network throughput while maintaining time fairness among all stations. The highest throughput gain can be obtained by disallowing transmissions from slow bitrate stations. Nonetheless, such throughput gain is not desirable because it is not fair.

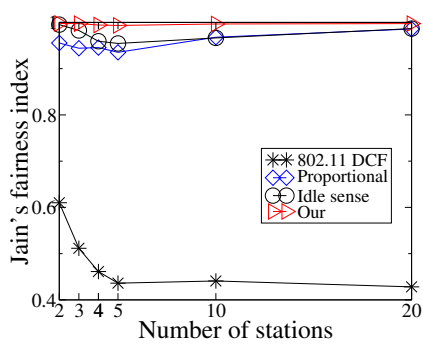

(a) Scenario I of $11 \mathrm{~b}$ mode.

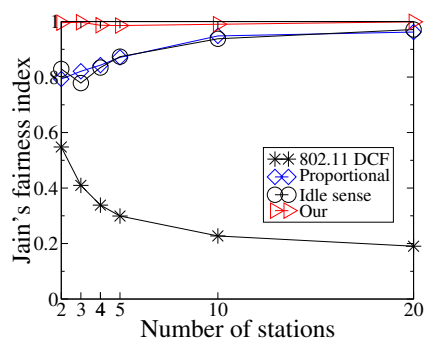

(c) Scenario I of $11 \mathrm{~b} / \mathrm{g}$ mode.

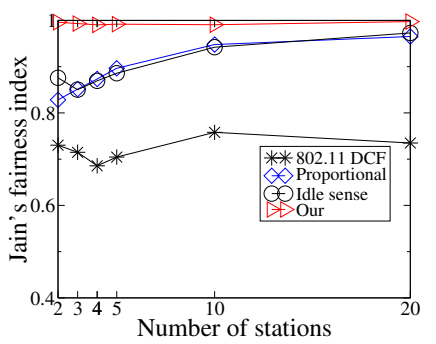

(b) Scenario I of $11 \mathrm{~g}$ mode.

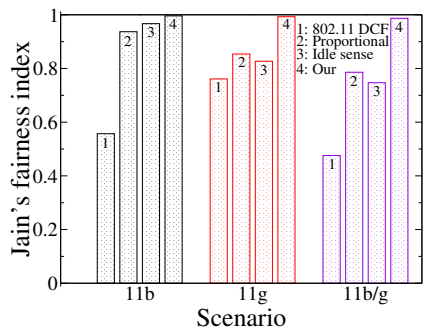

(d) Scenario II of all modes.
Fig. 1. Jain's fairness index.

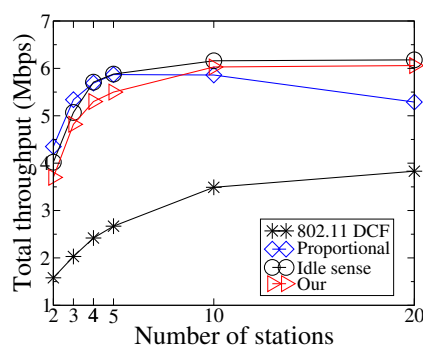

(a) Scenario I of $11 \mathrm{~b}$ mode.

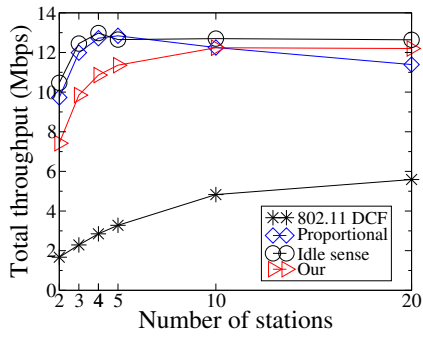

(c) Scenario I of $11 \mathrm{~b} / \mathrm{g}$ mode.

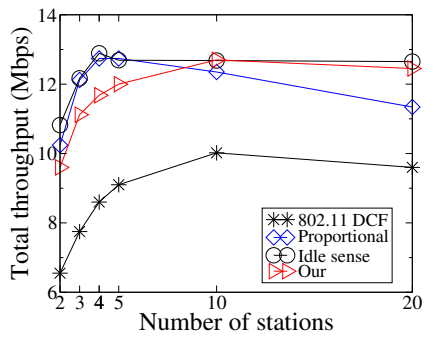

(b) Scenario I of $11 \mathrm{~g}$ mode.

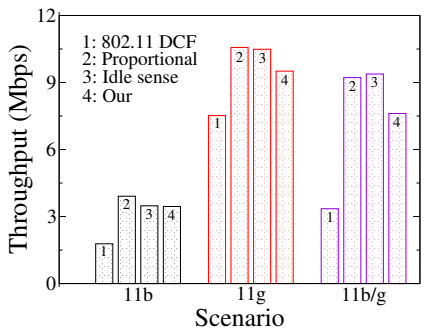

(d) Scenario II of all modes.
Fig. 2. Throughput.

Figure 2 presents the comparison of the total network throughput of the four algorithms. We can see that our proposed algorithm significantly outperforms the default 802.11 DCF scheme. Such great throughput improvement of our proposed algorithm is achieved via providing fair channel access time to all stations. We also notice that Idle Sense and Proportional achieve similar throughput to that of our proposed algorithm in most cases. However, please note that the throughput gain of Idle Sense and Proportional is obtained at the cost of scarifying fairness as it is shown in Figure 1.

3) Collision Overhead: We measure the collision overhead according to the ratio of total collisions experienced by the 
default 802.11 DCF scheme to those of the other three algorithms, denoted as collision ratio. The number of total collisions is acquired by summing all the collisions recorded by each station for each algorithm. It is necessary to point out that we skip the case of two competing stations because neither of the two stations is able to report collisions properly. We report the collision ratios among the four algorithms in Figure 3.

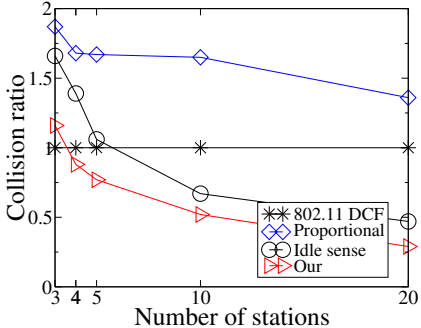

(a) Scenario I of $11 \mathrm{~b}$ mode.

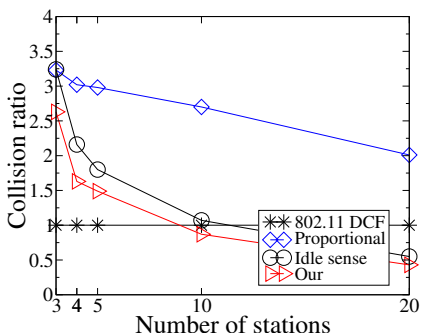

(c) Scenario I of $11 \mathrm{~b} / \mathrm{g}$ mode.

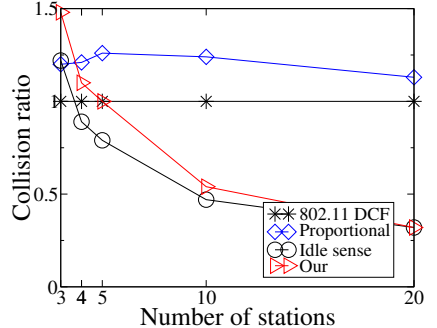

(b) Scenario I of $11 \mathrm{~g}$ mode.

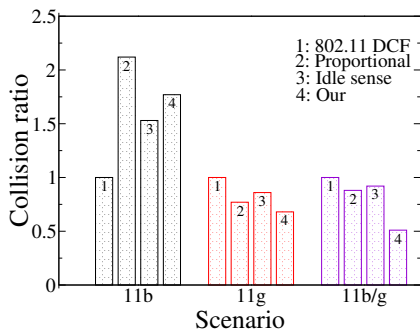

(d) Scenario II of all modes.
Fig. 3. Collision overhead.

The horizontal straight line with the collision ratio being 1 represents the default 802.11 DCF algorithm. Therefore, a curve below this line represents a decrease of collision overhead, while a curve above this line depicts an increase of collision overhead. We notice that the default 802.11 DCF scheme introduces the least collision overhead when the number of competing stations are small (i.e., less than 5). This seemingly anti-intuitive result is due to the fact that the other three algorithms transmit up to 5 times more frames than those of the default 802.11 DCF scheme (as it is shown in Figure 2). When the number of competing stations exceeds 5, both Idle Sense and our proposed algorithm start introducing lower collision overhead compared to that of the default 802.11 DCF scheme. We also notice that our proposed algorithm incurs lower collision overhead compared to that of Idle Sense and Proportional for most cases.

In summary, the evaluation results demonstrate that our proposed algorithm outperforms the other three algorithms in the following aspects: i) The channel occupancy time is almost equally shared (i.e., the Jain's fairness index approaching one) among all contending stations in each scenario; ii) Our proposed algorithm is able to maximize the aggregate throughput under the time fairness constraint; iii) Our algorithm is capable of greatly reducing collision overhead when the network exhibits certain complexities such as a richer bitrate diversity with a larger number of contending stations.

\section{CONCLUSION}

In this paper, we propose a novel MAC algorithm for multirate wireless LANs to maximize the aggregate throughput while maintaining time fairness among contending stations. Our proposed algorithm achieves these two objectives via letting each station select an appropriate contention window size in distributed manner. We evaluate the performance of our algorithm in a comprehensive comparative evaluation study. The evaluation results demonstrate that our proposed algorithm greatly outperforms three other popular MAC algorithms in the literature. As a part of our future work, we plan to implement our proposed algorithm in commodity 802.11 hardware and study its performance using real world experiments.

\section{REFERENCES}

[1] R. Jain, D. Chui, and W. Hawe, "A quantitative measure of fairness and discrimination for resource allocation in shared systems," Digital Equipment Institution, Tech. Rep., 1984.

[2] IEEE 802.11: Wireless LAN Medium Access Control (MAC) and Physical Layer (PHY) Specifications, IEEE, 2007.

[3] G. Bianchi, "Performance analysis of the ieee 802.11 distributed coordination function," IEEE Journal on Selected Areas in Communications, vol. 18 , no. 3, pp. 535-547, mar 2000.

[4] G. Tan and J. Guttag, "Time-based fairness improves performance in multi-rate wlans," in ATEC '04: Proceedings of the annual conference on USENIX Annual Technical Conference. Berkeley, CA, USA: USENIX Association, 2004.

[5] M. Heusse, F. Rousseau, G. Berger-Sabbatel, and A. Duda, "Performance anomaly of $802.11 \mathrm{~b}$," in INFOCOM '03: Proceedings of the International Conference on Computer Communications, vol. 2. IEEE Press, 2003, pp. 836-843.

[6] A. Banchs, P. Serrano, and H. Oliver, "Proportional fair throughput allocation in multirate ieee 802.11e wireless lans," Wirel. Netw., vol. 13, no. 5, pp. 649-662, 2007.

[7] M. Heusse, F. Rousseau, R. Guillier, and A. Duda, "Idle Sense: an optimal access method for high throughput and fairness in rate diverse wireless lans,' in SIGCOMM '05. New York, NY, USA: ACM, 2005, pp. 121-132.

[8] A. Miu, G. Tan, H. Balakrishnan, and J. Apostolopoulos, "Divert: finegrained path selection for wireless lans," in MobiSys '04: Proceedings of the 2nd international conference on Mobile systems, applications, and services, 2004, pp. 203-216.

[9] D. Kotz, C. Newport, R. S. Gray, J. Liu, Y. Yuan, and C. Elliott, "Experimental evaluation of wireless simulation assumptions," in MSWiM '04: Proceedings of the 7th ACM international symposium on Modeling, analysis and simulation of wireless and mobile systems. New York, NY, USA: ACM, 2004, pp. 78-82.

[10] Y. Jian and S. Chen, "Can CSMA/CA networks be made fair?" in MobiCom '08: Proceedings of the 14th ACM international conference on Mobile computing and networking. New York, NY, USA: ACM, 2008, pp. 235-246.

[11] G. Bianchi and I. Tinnirello, "Kalman filter estimation of the number of competing terminals in an ieee 802.11 network," in INFOCOM '03: Proceedings of the International Conference on Computer Communications, 2003.

[12] F. Calì, M. Conti, and E. Gregori, "Dynamic tuning of the ieee 802.11 protocol to achieve a theoretical throughput limit," IEEE/ACM Trans. Netw., vol. 8, no. 6, pp. 785-799, 2000.

[13] Y. Kwon, Y. Fang, and H. Latchman, "A novel mac protocol with fast collision resolution for wireless lans," in INFOCOM '03: Proceedings of the International Conference on Computer Communications, 2003. pp. 793-807.

[14] I. Aad, Q. Ni, C. Barakat, and T. Turletti, "Enhancing ieee $802.11 \mathrm{mac}$ in congested environments," Comput. Commun., vol. 28, no. 14, pp. 1605-1617, 2005.

[15] L. E. Li, M. Pal, and Y. R. Yang, "Proportional fairness in multi-rate wireless LANs,' in INFOCOM '08: Proceedings of the International Conference on Computer Communications, Phoenix, AZ, Apr. 2008.

[16] “OMNeT++ home page," http://www.omnetpp.org. 